\title{
Técnica de Caldwell-Luc en los últimos 16 años: Revisión de sus indicaciones
}

\author{
The Caldwell-Luc procedure in the last 16 years: \\ A review of its clinical indications
}

Hayo Breinbauer K¹, José Miguel Contreras R², Carlos Namoncura P².

\begin{abstract}
RESUMEN
Introducción: En una época marcada por el abordaje endoscópico del seno maxilar, principalmente en casos de sinusitis crónica, la técnica clásica de Caldwell-Luc ha quedado relegada a una situación casi de 'recuerdo del pasado'. Sin embargo, evidencias recientes Ilaman a rescatar la utilidad de este procedimiento frente a fracasos de cirugías endoscópicas funcionales sinusales, especialmente relacionadas con una entidad conocida como 'cambios irreversibles de la mucosa'.

Objetivo: Reflexionar sobre las indicaciones de esta técnica en nuestro centro, comparándolas con las nuevas perspectivas esbozadas en la literatura mundial.

Material y Método: Se presenta una revisión de la literatura al respecto, junto con la casuística del Servicio de Otorrinolaringología del Hospital San Juan de Dios.

Resultado: Entre 1992 y 2007 se realizaron 56 cirugías Caldwell-Luc. La principal indicación fue la resolución de sinusitis crónica maxilar, aunque en relación a situaciones que se alejan de los motivos propuestos en la literatura para elegir este procedimiento. Esto, junto a una alta tasa de recidiva sintomática (21,4\% de los pacientes operados entre 1997 y 2007 cumplen criterios para sinusitis crónica actual) y elevadas tasas de complicaciones $(31,8 \%)$ nos llevan a reflexionar sobre el uso que se ha dado a esta técnica en nuestro centro.

Conclusión: Se concluye que este procedimiento continúa vigente, y quizás siendo irremplazable, especialmente en el manejo de patología sinusal crónica con cambios irreversibles en su mucosa, concepto sin una definición precisa y sobre el cual comentamos en detalle.

Palabras clave: Caldwell-Luc, sinusitis maxilar, senos paranasales, cambio irreversible de mucosa
\end{abstract}

\section{ABSTRACT}

Introduction. Since the introduction of the endoscopic approach to the maxillary sinus, Caldwell-Luc's classic technique has been relegated to an almost 'memory of the past' category. Yet, recent evidence urges to rescue this procedure in view of its usefulness in cases of failed functional endoscopic sinus surgery, especially related to an entity known as 'irreversible mucosa changes'.

Aim. To review the indications for this technique in our department, comparing our data with that from recent literature.

Médico-Cirujano, Universidad de Chile.

2 Médico del Servicio de Otorrinolaringología, Hospital San Juan de Dios, Campus Occidente, Universidad de Chile. 
Results. We present 56 Caldwell-Luc procedures and a literature review on the subject. The procedures were performed between 1992 and 2007 at the ENT Department of San Juan de Dios Hospital. Chronic sinusitis remained the main indication, but within scenarios that differ from those regarded in the current literature as indicative of the technique. This, along with a large symptomatic relapse rate (21.4\% of patients operated between 1997 and 2007 met criteria for current chronic sinusitis), and high complications rate (31.8\%) within our cases, lead us to question the indications for the procedure in our department.

Conclusion. We conclude that this procedure remains in force, and is perhaps even irreplaceable, particularly in the management of chronic sinus disease with irreversible mucosa changes, an ill-defined concept, which we discuss in detail.

Key Words: Caldwell-Luc, maxillary sinus, paranasal sinuses, irreversible mucosa changes.

\section{INTRODUCCIÓN}

El abordaje de las cavidades paranasales ha experimentado un enorme avance en las últimas décadas, constituyendo durante este período una verdadera revolución la implementación de las técnicas endoscópicas. Como en otras disciplinas quirúrgicas, estos procedimientos mínimamente invasivos han desplazado a las técnicas clásicas, las cuales han quedado relegadas a una situación de 'recuerdos del pasado'. Particularmente en el abordaje del seno maxilar, la cirugía endoscópica funcional sinusal (CEFS) prácticamente ha reemplazado a la técnica de Caldwell-Luc (CL). Sin embargo, evidencias recientes llaman a rescatar esta cirugía abierta, destacando su utilidad en situaciones específicas donde procedimientos endoscópicos mínimamente invasivos aún no tienen resultados satisfactorios.

Esta técnica fue descrita en 1893 por George Caldwell (U.S.A.), y un año más tarde por Guy Luc (Francia). También lo hizo Spicer (Inglaterra) aunque su nombre no trascendió en la historia. Consiste en el abordaje del seno maxilar por vía vestibular a través de la fosa canina. La entrada al seno puede ser realizada con martillo y cincel, mediante uso de fresa, o utilizando un trócar en la punción inicial para luego abrir la ventana ósea con fórceps de Kerrison ${ }^{1}$. Así se logra una excelente visión del seno, lo que permite remover completamente su mucosa lo que, como se verá más adelante, constituye la principal ventaja de esta técnica. Clásicamente se realizaba una antrostomía inferior cuya función, según el concepto original de Caldwell, era la de permitir el lavado y el manejo posoperatorio inmediato. Posteriormente se con- templó como medida de drenaje gravitacional de la cavidad. Sin embargo, se ha descrito que hasta $82 \%$ de las antrostomías inferiores están cerradas a los 3 meses $^{2}$, y se ha demostrado que la dirección del drenaje mucociliar persiste hacia el ostium natural en el meato medio ${ }^{3}$. Por ende, se recomienda en la actualidad sólo realizar antrostomía en el meato medio ${ }^{4}$.

Antes de la llegada de la cirugía endoscópica, a finales de los 70', la indicación clásica para la técnica $\mathrm{CL}$ era la sinusitis crónica maxilar que no respondía a tratamiento médico. En los 80' y los 90', fue reemplazada ampliamente en este rol por las CEFS, y sólo en contadas ocasiones una sinusitis crónica era tratada con esta técnica clásica. Para el procedimiento de CL persistieron sólo otras indicaciones, mucho menos frecuentes, las así llamadas "extra-sinusitis". Estas incluyen la descompresión del exoftalmo en la enfermedad de Basedow-Graves, el abordaje del espacio ptérigomaxilar, fístulas oro-antrales, traumas faciales, cuerpos extraños, lesiones odontogénicas, ósteonecrosis, bola fúngica y neoplasias ${ }^{5,6}$.

Sin embargo, transcurridos 20 años de era endoscópica, una revisión del año 2003 menciona nuevamente el rol de la técnica $C L$ en sinusitis crónica, pero en relación con CEFS previa fracasa$\mathrm{da}^{7}$. En un análisis retrospectivo de $62 \mathrm{CL}$ en 2005 , el $46,7 \%$ de los pacientes tuvo como diagnóstico una sinusitis maxilar crónica. De éstos, más de la mitad presentaba una CEFS previa fracasada. En los pacientes restantes, al sospecharse 'cambios irreversibles de la mucosa', se planteó que una CEFS no resultaría beneficiosa ${ }^{6}$.

Esta nueva posición, que parece estar adquiriendo la técnica $\mathrm{CL}$, es entendible debido a la 
fisiopatología de la sinusitis crónica maxilar. El abordaje de la CEFS se basa en el principio de la obstrucción del complejo osteomeatal como causa fundamental de esta patología. Sin embargo, reducir exclusivamente a este factor la patogénesis de la sinusitis crónica, parece una excesiva simplificación $^{8}$. Un segundo aspecto relevante consiste en los cambios que sufre la mucosa del seno maxilar, los que pueden adquirir el carácter de irreversible, y donde la única solución parece ser la remoción radical de este epitelio ${ }^{9}$.

El abordaje abierto y la posibilidad de extirpar completamente la mucosa, características de la técnica $\mathrm{CL}$, adquieren en este escenario toda su utilidad. Tanto en investigaciones en animales como en humanos, el epitelio del seno maxilar ha mostrado gran capacidad para regenerarse tras una cirugía $\mathrm{CL}$, a pesar de una conocida remodelación ósea subyacente. Aunque esta mucosa no alcanza un estado de normalidad, logra una mejoría significativa, la que -en generalpermite el manejo sintomático con terapia médi$\mathrm{Ca}^{10-13}$. En un estudio realizado en humanos, la disminución de las características inflamatorias del epitelio maxilar mostró ser significativamente mayor tras una técnica $\mathrm{CL}$, que luego de una CEFS ${ }^{14}$.

A pesar de estas ventajas, no se recomendó la cirugía CL como tratamiento de primera línea en sinusitis maxilar, principalmente debido a una alta tasa de complicaciones consideradas casi inevitables $\left(10 \%\right.$ al $\left.40 \%{ }^{5}\right)$. Una revisión de 670 procedimientos CL informa de $19 \%$ de complicaciones, siendo la más frecuente la lesión del nervio infraorbitario $(9,1 \%)$, seguidas por dacriocistitis, fístula oro-antral, asimetría facial y desvitalización dental15. Sin embargo, trabajos recientes señalan que las complicaciones serían en gran medida dependientes de la experiencia y el cuidado operatorio del cirujano. Destaca la práctica de un cirujano avezado, quien en 133 casos sólo observó complicaciones en 4 pacientes. En todos hubo una lesión del nervio infraorbitario, dos de los cuales se recuperaron espontáneamente a los 3 meses de evolución ${ }^{7}$. En la técnica quirúrgica tendrían importancia para reducir el número de complicaciones: una entrada cuidadosa al seno, la protección del nervio infraorbitario durante la elevación del periostio, y el cierre cuidadoso de éste.

Aunque existe acuerdo en que la CEFS es el tratamiento quirúrgico inicial en sinusitis maxilar cróni- ca, ésta tendría un resultado efectivo sólo entre el 74\% y $97 \%$ de los casos $^{16-18}$. Ante la persistencia sintomática y la necesidad de una ulterior cirugía de revisión se ha acuñado el concepto de 'CEFS fracasada'. Las causas de estos fracasos son variadas e incluyen la presencia de poliposis, enfermedad sinusal extensa, alteraciones anatómicas, factores ambientales (vgr: exposición a alérgenos, tabaco, moho, etc.); del huésped (asma, intolerancia a la aspirina, inmunodeficiencias, enfermedades granulomatosas, disquinesia ciliar primaria, fibrosis quística, etc.), e iatrogenia en la cirugía previa (vgr: lateralización del cornete medio y formación de sinequias posoperatorias, las que obstruirían nuevamente el ostium del seno maxilar) ${ }^{19,20}$. Sin embargo, cuando no se logra demostrar alguna de estas causas, y existe un ostiumpermeable, la falla de la CEFS estaría en relación con el concepto de 'cambios irreversibles de la mucosa $^{21}$. Esta sería, en frecuencia, la quinta causa de fracaso de la CEFS. Estos pacientes se beneficiarían con la extirpación completa de la mucosa sinusal mediante una técnica $\mathrm{CL}$, esperándose la posterior regeneración del epitelio, y una subsecuente mejoría sintomática. Incluso hay quienes proponen que, al disponer de evidencias preoperatorias de cambios irreversibles de la mucosa, se podría considerar un abordaje radical del seno como tratamiento inicial ${ }^{9}$.

\section{MATERIAL Y MÉTODO}

Con el fin de reflexionar sobre las indicaciones y la situación de esta técnica en nuestro centro, comparándola con las nuevas perspectivas esbozadas en la literatura internacional, quisimos revisar la casuística en los últimos 16 años del Servicio de Otorrinolaringología del Hospital San Juan de Dios en la técnica CL y abordaje de cavidades paranasales.

Se revisó el archivo quirúrgico de dicho centro entre enero de 1992 y diciembre de 2007. A la totalidad de los pacientes operados mediante técnica $\mathrm{CL}$ que se logró contactar, se le aplicó la encuesta estandarizada para evaluación de síntomas nasosinusales e impacto en calidad de vida SNOT-2023-25. Esta consiste en 20 indicadores, cada uno de los cuales recibe una calificación de 0 a 5 por parte del paciente, según el nivel de molestia que un determinado síntoma le produce. A los pacientes que pudieron asistir al policlínico se les realizó una nasofaringolaringoscopía. 


\section{RESULTADOS}

Se encontraron 424 procedimientos en cavidades paranasales, lo que representó $3 \%$ de un total de 14.102 cirugías realizadas durante el período revisado. De éstos, $57 \%$ se efectuó por vía endoscópica. El resto consideró técnicas no endoscópicas como técnica $\mathrm{CL}$, osteoplastías frontales, operaciones de Lynch 0 de Riedel y polipectomías nasales, entre otras. Se practicaron 56 cirugías CL (constituyó el $29 \%$ de los procedimientos no endoscópicos) en 52 pacientes (4 de ellos bilaterales). En la Figura $1 \mathrm{se}$ presenta el número por año de CEFS y de cirugías no endoscópicas en cavidades paranasales, a lo largo de los 16 años analizados. Dentro de estas últimas, los procedimientos $\mathrm{CL}$ se muestran en línea discontinua. La primera cirugía endoscópica se llevó a cabo en mayo de 1994. Sin embargo, el número de CEFS fue relativamente bajo hasta 1997, luego de lo cual se advierte un importante aumento en la realización de este procedimiento, superando a los procedimientos no-endoscópicos, los que durante ese mismo período se redujeron en forma significativa.

Para efectos de este trabajo hemos definido arbitrariamente dos períodos: a) un período 'preendoscópico' entre 1992 y 1996, durante el cual se realizaron 30 cirugías $\mathrm{CL}$ (con un promedio de 6 anuales), y b) un período 'endoscópico' entre 1997 y 2007 con 26 casos (promedio de 2,4 anuales). Destaca, especialmente en este último período, un nivel relativamente constante en la utilización de la técnica CL a lo largo de los años (Figura 1).

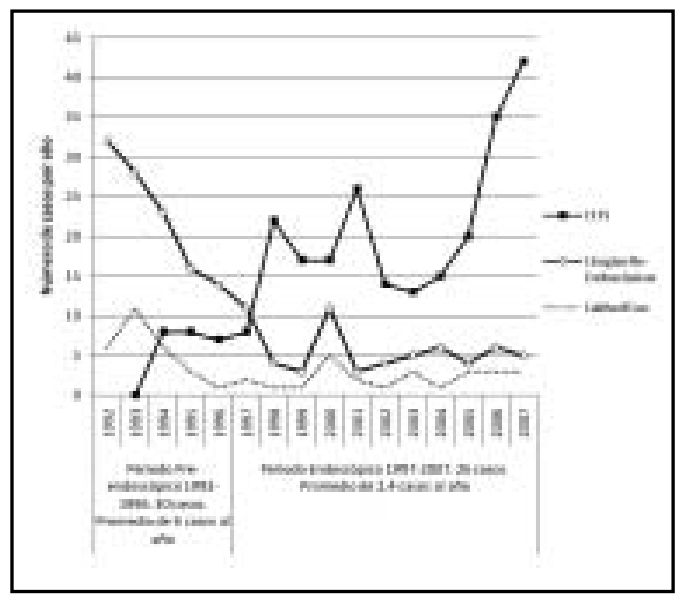

Figura 1. Distribución en el tiempo de cirugías en cavidades paranasales.
Debido a los cambios en las apreciaciones sobre la técnica CL desde la aparición de la CEFS, decidimos concentrar nuestro análisis en las 26 cirugías CL (en 25 pacientes) durante el período endoscópico. En este grupo se revisaron 22 fichas ( $88 \%$ de los casos del período), se efectuó evaluación sintomática a 15 pacientes $(60 \%)$ y se practicó una nasofaringolaringoscopía a 12 pacientes (48\%). En el período preendoscópico (30 casos de cirugías CL) se utilizó la información registrada en el archivo quirúrgico (diagnóstico y procedimiento realizados), se encuestó a 10 pacientes $(33,3 \%$ ) y se realizó una nasofaringolaringoscopía en 6 de ellos (20\%). Lo anterior correspondió a la totalidad de pacientes que fue posible ubicar en base a los registros del hospital.

En la Figura 2 se aprecia la repartición de los diagnósticos de los 56 casos de cirugías CL, agrupados según período analizado. Es posible observar una distribución relativamente semejante entre ambos períodos, donde destaca el predominio que tiene como diagnóstico la sinusitis crónica.

Al profundizar en los factores que motivaron la realización de una técnica $\mathrm{CL}$ en los casos con diagnóstico de sinusitis crónica maxilar, en la Figura 3 se observa que hasta el año 1996 el 83\% de estas cirugías se llevó a cabo por sinusitis crónica. Durante el período endoscópico, sin embargo, esta cifra sólo alcanzó el $32 \%$, adquiriendo mayor importancia el origen de la sinusitis en un foco dental y la sospecha de una bola fúngica. Destaca en este período que casi una cuarta parte de los pacientes fueron operados mediante cirugía $\mathrm{CL}$ al no poder realizarse una CEFS, ya fuese por alteraciones anatómicas, sangrado abundante, o por imposibilidades técnicas en el acto quirúrgico. El 14\% de las técnicas CL realizadas entre 1997 y 2007 tuvieron una CEFS previa que puede calificarse como fracasada, y Ilama la atención que $23 \%$ de los casos durante este período haya consistido en la necesidad de convertir una CEFS en un ulterior Caldwell-Luc.

En cuanto a las complicaciones se obtuvo una tasa global de $31,8 \%$, la que si bien se ubica dentro de los rangos descritos en la literatura, se considera relativamente alta. La principal complicación fue la parestesia de la región del nervio infraorbitario $(27,3 \%)$, seguida de dacriocistitis $(4,5 \%)$.

La encuesta SNOT-20 permite un análisis global del impacto de síntomas nasosinusales en la 


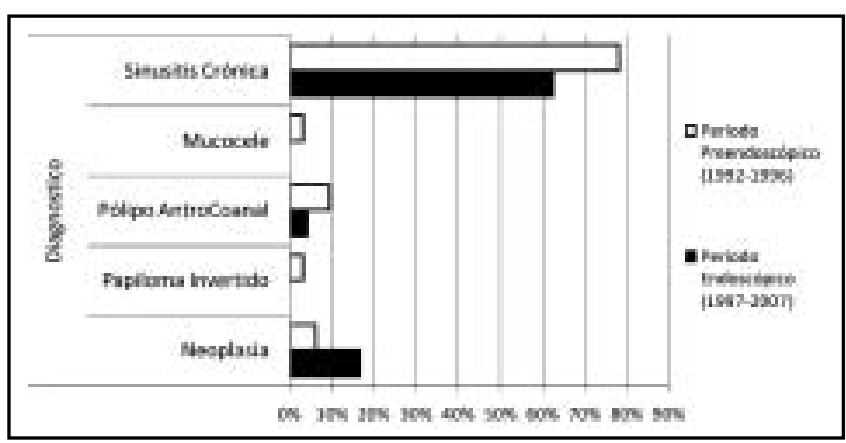

Figura 2. Distribución de cirugías Caldwell-Luc según diagnóstico y período.

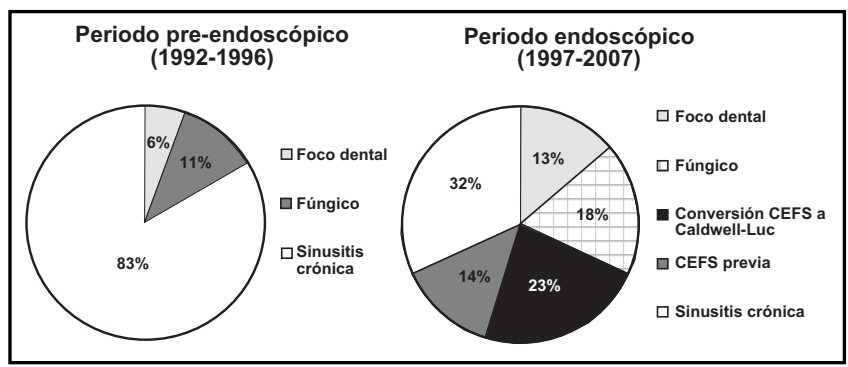

Figura 3. Motivos para uso de la técnica Caldwell-Luc en sinusitis crónica según período.

calidad de vida del enfermo en una escala de 0 a 100 puntos, donde una persona sana tendría 0 punto. Los pacientes operados en el período endoscópico (1997-2007) a los que fue posible evaluar $(60 \%$ de ellos), tenían en promedio 25,5 puntos en esta escala (con un rango de 5 a 56 puntos). Al desglosar los 20 indicadores de la encuesta, encontramos que $42,9 \%$ de los pacientes declaró tener dolor facial al momento de esta evaluación, 46,7\% presentaba secreción nasal abundante y $26,7 \%$ refirió sensación de descarga posterior. En este grupo, el 21,4\% de los pacientes encuestados cumplía con los criterios actuales para sinusitis crónica.

Aunque el número de casos a los que fue posible evaluar en el período 1992-1996 fue escaso (10 pacientes, esto es $33,3 \%$ del total) se encontró una diferencia estadísticamente significativa $(p<0,05)$ al comparar el promedio de su puntuación (13,4 puntos) en la escala SNOT-20 con la del grupo operado entre 1997 y 2007 (25,5 puntos).

Al examen mediante nasofaringolaringoscopía se pudo observar que de los pacientes en quienes se había realizado una antrostomía media, $83,3 \%$ de ellas permanecía permeable. En quienes se practicó una antrostomía inferior, el $44,4 \%$ de éstas se mantenía abierta. De tres pacientes en los que se había efectuado ambas antrostomías, éstas permanecían abiertas sólo en un caso.

\section{DISCUSIÓN}

La casuística analizada de cirugías CL realizadas durante el período endoscópico (1997-2007), revela una elevada tasa de complicaciones, una importante recidiva de síntomas nasosinusales (más del $20 \%$ de los pacientes cumple con criterios para sinusitis crónica actual), y un importante deterioro en la calidad de vida secundario a estos síntomas. Más aún, en términos de calidad de vida y recidiva sintomática el resultado de este grupo es significativamente peor respecto a los pacientes operados mediante técnica $\mathrm{CL}$ antes de la instauración de la cirugía endoscópica.

Lo anterior obliga a cuestionarnos el uso que se ha dado a esta técnica en los últimos años.

Considerando que el instrumento de evaluación utilizado mide calidad de vida, es posible pensar que los peores resultados encontrados en el último período se deban a pacientes con mayores expectativas y una actitud más crítica respecto a décadas pasadas. También es concebible que los pacientes operados en el período endoscópico hayan presentado una mayor complejidad, y en quienes no se planteó una CEFS por considerarla incapaz de resolver el problema. Por otro lado, al utilizarse la técnica CL menos frecuentemente, se puede asumir que existe un menor entrenamiento en ella, con un impacto en su efectividad y en las complicaciones.

Por último, este análisis nos lleva a considerar la posibilidad que la indicación de nuestros CL no haya sido la óptima. Si comparamos los motivos registrados en los casos revisados, para realizar un $C L$ con los de la literatura encontramos importan- 
tes diferencias. En la actualidad se plantea que el origen en un foco dental y el diagnóstico de bola fúngica debiesen ser perfectamente abordados por vía endoscópica ${ }^{26-28}$. Además, se cuestiona el abordaje mediante técnica $\mathrm{CL}$ en pacientes con neoplasias malignas por favorecer la contaminación tumoral hacia la pared anterior y vestíbulobucal, siendo recomendada la vía endoscópica para realizar biopsias e, incluso, extirpación de tumores en seno maxilar ${ }^{29}$. Asimismo la conversión de CEFS a una cirugía CL estaría más relacionada con la falta de dominio en la técnica quirúrgica (la que debiese ser el tratamiento de primera línea) que en una indicación formal y avalada de una técnica CL.

Las indicaciones para ésta, avaladas en la literatura actual, corresponderían principalmente a CEFS fracasadas, especialmente aquéllas relacionadas con cambios irreversibles de la mucosa. Persisten, además, algunas recomendaciones clásicas 'extra-sinusitis', considerando que la descompresión del exoftalmo en la enfermedad de Basedow Graves, el abordaje del espacio ptérigomaxilar y algunos cuerpos extraños pudiesen ser resueltos actualmente por vía endoscópica ${ }^{30-35}$.

El concepto de cambios irreversibles de la mucosa se define como la falla en el aparato mucociliar, lo que se demuestra en la práctica por una enfermedad sinusal persistente después de tratamientos médicos máximos y con presencia de una adecuada antrostomía media ${ }^{22}$. Sin embargo, no hay acuerdo sobre una definición histológica 0 de criterios imagenológicos que describan esta entidad, ni de medios que permitan pronosticarla (o de predecir el fracaso de una CEFS) ${ }^{36-40}$.

La biopsia del proceso uncinado efectuada durante una CEFS ha permitido esbozar algunos parámetros histológicos, como un mayor número de células caliciformes, la pérdida de cilios, el engrosamiento de la membrana basal y la infiltración de mastocitos y eosinófilos ${ }^{36}$. Cada uno de estos indicadores se vincularía con la persistencia, posterior a una CEFS, de algunos síntomas específicos. Así, por ejemplo, un mayor número de células caliciformes se relaciona con la persistencia de congestión, secreción nasal y cefalea. Sin embargo, ninguno de estos criterios -individual 0 conjuntamente- predicen un fracaso en la CEFS o permiten definir cambios irreversibles.
En términos imagenológicos las clasificaciones actuales (Kennedy, Lund-McKay) hablan más de severidad global y de obstrucción del complejo osteomeatal que de características específicas del seno que puedan asociarse a cambios irreversibles y, por sí solas, tampoco predicen el fracaso de la $\mathrm{CEFS}^{38}$. Se encuentra aún pendiente el análisis de imágenes de remodelación ósea y su relación con otros criterios histológicos y clínicos.

\section{CONCLUSIONES}

La literatura y los casos quirúrgicos que se han revisado muestran claramente un predominio de las técnicas endoscópicas en el manejo del seno maxilar, especialmente en el caso de sinusitis crónica. Sin embargo se sigue realizando cirugías CL, aunque a veces sin una indicación adecuada y, en general, en casos donde de manera intuitiva se sospecha que la CEFS no tendría un resultado óptimo. Esta situación de poca claridad en cuanto a su verdadera utilidad, posiblemente ha condicionado un deterioro en los resultados clínicos de este versátil procedimiento. La evidencia reciente apunta a la existencia de un escenario específico en CEFS fracasadas con ostium permeable y sin causas identificables, donde la extirpación radical de la mucosa parece ser indispensable.

Por consiguiente, la cirugía CL es una técnica vigente y necesaria en la actualidad, marcada en estos tiempos por el abordaje endoscópico del seno maxilar, y su indicación estaría principalmente relacionada con CEFS fracasadas y al concepto de cambios irreversibles de la mucosa. Urge profundizar nuestro conocimiento sobre esta entidad, buscando definir factores que ayuden a predecir tanto pacientes con mayor riesgo de fracaso de una CEFS como situaciones en las cuales una técnica $\mathrm{CL}$ debiese practicarse posterior a dicho fracaso 0 , incluso, como un tratamiento de primera línea.

\section{BIBLIOGRAFÍA}

1. MacBeth R. Caldwell Luc and their operation. Laryngoscope 1971; 81: 1652-7.

2. Linuma T, Oosama H, Haruyama K, et al. Morphology of the inferior nasal meatus. Pract Otol Kyoto 1982; 75: 931-5. 
3. Coleman JR, Duncavage Ja. Extended middle meatal antrostomy: The treatment of circular flow. Laryngoscope 1996; 106: 1214-7.

4. Al-Belasy FA. Inferior meatal antrostomy: Is it necessary after radical sinus surgery through the Caldwell-Luc approach? J Oral Maxillofac Surg 2004; 62: 559-62.

5. Blitzer A, Lawson W. The Caldwell Luc procedure in 1991. Oolaryngol Head Neck Surg 1991; 105: 717-22.

6. Barzilai G, Greenberg E, Uri N. Indications for the Caldwell-Luc approach in the endoscopic era. Aolaryngol Head Neck Surg 2005; 132: 219-20.

7. Matheny K, Duncavage J. Contemporary indications for the Caldwell-Luc procedure. Ourr Opin Aolaryngol Head Neck Surg 2003; 11: 22-6.

8. Salman SD. The abnormal ostiomeatal complex and sinusitis. Am J Rhinol 1992; 6: 29-32.

9. El-Fattah R, Nour Y, El-Daly A. Endoscopic radical antrectomy: a permanent replacement for the Caldwell-Luc operation. J Laryngol Otol 2008; 122: 268-76.

10. GORHAM CB, BACHER JA. Regeneration of the human maxillar antral lining. Arch Oolaryngol 1930; 11: 763-71.

11. Benniger MS, Sebek BA, Levine HL. Mucosal regeneration of the maxillary sinus after surgery. Otolaryngol Head Neck Surg 1989; 101: 33-7.

12. Kennedy DW, Shatan $H$. Reevaluation of maxillary sinus surgery: experimental study on rabbits. Ann Otol Rhinol Laryngol 1989; 98: 901-6.

13. Forsgren K, Stierna P, Kumlien J, Fukami M, PentTILa M. Regeneration of the maxillary sinus mucosa following surgical removal. Experimental study on rabbits. Ann Otol Rhinol Laryngol 1993; 102: 459-66.

14. Forsgren K, Fukami M, Penttila M, Kumlien J, Stierna P. Endoscopic and Caldwell-Luc approaches in chronic maxillary sinusitis: A comparative histopathologic study on preoperative and postoperative mucosal morphology. Ann Col Rhinol Laryngol 1995; 104: 350-7.

15. DeFreitas J, Lucente F. The Caldwell-Luc procedure: Institutional review of 670 cases: 1975-1985. Laryngoscope 1988; 98: 1297-300.
16. Battacharyy N. Clinical outcomes after revision endoscopic sinus surgery. Arch Otolaryngol Head Neck Surg 2004; 130: 975-8.

17. Levine HL. Functional endoscopic sinus surgery: evaluation, surgery, and follow-up of 250 patients. Laryngoscope 1990; 100: 79-84.

18. KENNEDY DW. Prognostic factors, outcomes and staging in ethmoid sinus surgery. Laryngoscope 1992; 102(Suppl 57): 1-18.

19. CHow J. Technical reasons for endoscopic sinus surgery failures. Ourr Opin Otolaryngol Head Neck Surg 2002; 10(1): 33-5.

20. Cohen N, Kennedy D. Endoscopic sinus surgery: Where we are and where we're going. Ourr Opin Aolaryngol Head Neck Surg 2005; 13: 32-8.

21. Cutler Jl, Duncavage JA, Matheny K, Cross JL, Miman MC, OH CK. Results of Caldwell LuC after failed endoscopic middle meatus antrostomy. Laryngoscope 2003; 113: 214850.

22. Richtsmeier WJ. Top 10 reasons for endoscopic maxillary sinus surgery failure. Laryngoscope 2001; 111(11): 1952-6.

23. Van Oene C, Relu E, Sprangers M et al. Qualityassessment of disease-specific quality of life questionnaires for rhinitis and rhinosinusitis: A systematic review. Allergy 2007; 62: 1359-71.

24. Morley A.D., Sharp H.R. (2006) A review of sinonasal outcome scoring systems - which is best? Cin Aolaryngol 2006; 31(2): 103-9.

25. Piccirillo J, Merrit M, Richards M. Psychometric and clinimetric validity of the 20-Item Sino-Nasal Outcome Test (SNOT-20). Otolaryngol Head Neck Surg 2002; 126: 41-7.

26. Pagella F, Matti E, DeBernardi F et al. Paranasal sinus fungal ball: Diagnosis and management. Mycoses 2007; 50(6): 451-6.

27. Grosjean P, Weber R. Fungus ball of the paranasal sinuses: A review. Eur Arch Corhinolaryngol 2007; 264(5): 461-70.

28. Costa F, Emanuelli E, Robiony M, Zerman N, PoLINI F, PoLITI M. Endoscopic surgical treatment of chronic maxillary sinusitis of dental origin. J Oral Maxillofac Surg 2007; 65(2): 223-8.

29. RICE DH. Endonasal approaches for sinonasal and nasopharyngeal tumors. Otolaryngol Cin North Am 2001; 34(6): 1087-93. 
30. Dubin MR, Tabaee A, Scruggs JT, Kazim M, Close LG. Image-guided endoscopic orbital decompression for Graves' orbitopathy. Ann Otol Rhinol Laryngol 2008; 117(3): 177-85.

31. Jurado-Ramos A, Romero FR, Baños EC, Molina JS. Minimally invasive endoscopic techniques for treating large, benign processes of the nose, paranasal sinus, and pterygomaxillary and infratemporal fossae: solitary fibrous tumor. $J$ Laryngol Ool 2008; 11: 1-5.

32. Awwad RJ, Goval P, Emko P. Endoscopic transnasal approach for retrieval of foreign bodies from the pterygomaxillary fossa. $\mathrm{Am} \mathrm{J}$ Aolaryngol 2006; 27(6): 440-2.

33. Pritikin JB, Caldarelli DD, Panje WR. Endoscopic ligation of the internal maxillary artery for treatment of intractable posterior epistaxis. Ann Otol Rhinol Laryngol 1998; 107(2): 85-91.

34. Lubbe DE, Aniruth S, Peck T, Liebenberg S. Endoscopic transnasal removal of migrated dental implants. Br Dent J2008; 204(8): 435-6.

35. Sugiura N, OchI K, Komatsuzaki Y. Endoscopic extraction of a foreign body from the maxillary sinus. Otolaryngol Head Neck Surg 2004; 130(2): 279-80.

36. Baudoin T, Cupic H, Geber G, VaGI_ D, GRGI_ M, KalogJera L. Histopathologic parameters as predictors of response to endoscopic sinus surgery in nonallergic patients with chronic rhinosinusitis. Otolaryngol Head Neck Surg 2006; 134: 761-6.

37. DRVI_ P, _uPI_ H, BaudoIN T, ET AL. Pathohistologic study on mucosal morphology of chronic maxillary sinusitis. Acta Cin Croat 2004; 43: 210-22.

38. Hopkins C, Browne J, Slack R, Lund V, Brown P. The Lund-Mackay staging system for chronic rhinosinusitis: How is it used and what does it predict? Aolaryngol Head Neck Surg 2007; 137: 555-61.

39. Bradley D, Kountakis S. Correlation between computer tomography scores and improvement after endoscopic sinus surgery. Laryngoscope 2005; 115: 466-9.

40. BHATtACHARYA N. Radiographic stage fails to predict symptom outcomes after endoscopic sinus surgery for chronic rhinosinusitis. Laryngoscope 2006; 116: 18-22.

41. BenNiger M. Endoscopic sinus surgery: what we know from literature. Ourr Opin Otolaryngol Head Neck Surg 2002; 10(1): 44-8.

42. Murray J, Jackson M. Complications after treatment of chronic maxillary sinus disease with Caldwell-Luc procedure. Laryngoscope1983, 93: 282-4.

43. Guo Y, Mauima Y, Hattori M et al. Effects of functional endoscopic sinus surgery on maxillary sinus mucosa. Arch Aolaryngol Head Neck Surg 1997; 123: 1097-100.

44. Damn M, Quante $G$, Jugehuelsing M. Impact of functional endoscopic sinus surgery on symptoms and quality of life in chronic rhinosinusitis. Laryngoscope 2002; 112: 31015.

45. Videler W, Wreesman V, van der Meulen F et al. Repetitive endoscopic sinus surgery failure: A role for radical surgery? Aolaryngol Head Neck Surg 2006; 134: 586-91.

46. Palmer JN, Kennedy DW. Medical management in functional endoscopic sinus surgery failures. Ourr Opin Atolaryngol Head Neck Surg 2003; 11: 6-12.

47. Cohen NA, Kennedy DW. Revision endoscopic sinus surgery. Otolaryngol Cin North Am 2006; 39: 417-35.

\footnotetext{
Dirección: Dr. Hayo Breinbauer K.

Walter Scott 1439, Vitacura. Santiago de Chile. E mail: hayo.bk@gmail.com
} 